\title{
An Astrophysical Peek into Einstein's Static Universe: No Dark Energy
}

\author{
Abhas Mitra \\ Theoretical Astrophysics Section, Bhabha Atomic Research Centre, Mumbai, India \\ Homi Bhabha National Institute, Mumbai, India \\ E-mail: amitra@barc.gov.in \\ Received June 26, 2011; revised August 28, 2011; accepted September 13, 2011
}

\begin{abstract}
It is shown that in order that the fluid pressure and acceleration are uniform and finite in Einstein's Static Universe (ESU), $\Lambda$, the cosmological constant, is zero. $\Lambda$ being a fundamental constant, should be the same everywhere including the Friedman model. Independent proofs show that it must be so. Accordingly, the supposed acceleration of the universe and the attendant concept of a "Dark Energy" (DE) could be an illusion; an artifact of explaining cosmological observations in terms of an oversimplified model which is fundamentally inappropriate. Indeed observations show that the actual universe is lumpy and inhomogeneous at the largest scales. Further in order that there is no preferred centre, such an inhomogeneity might be expressed in terms of infinite hierarchial fractals. Also, the recent finding that the Friedman model intrinsically corresponds to zero pressure (and hence zero temperature) in accordance with the fact that an ideal Hubble flow implies no collision, no randomness (Mitra, Astrophys. Sp. Sc., 333,351, 2011) too shows that the Friedman model cannot represent the real universe having pressure, temperature and radiation. Dark Energy might also be an artifact of the neglect of dust absorption of distant Type 1a supernovae coupled with likely evolution of supernovae luminosities or imprecise calibration of cosmic distance ladders or other systemetic errors (White, Rep. Prog. Phys., 70, 883, 2007). In reality, observations may not rule out an inhomogeneous static universe (Ellis, Gen. Rel. Grav. 9, 87, 1978), if the fundamental "constant"s are indeed constant.
\end{abstract}

Keywords: General Relativity, Cosmological Constant, Cosmology, Dark Energy, Fractal Cosmology, Static Universe, Big Bang Theory

\section{Introduction}

The "cosmological principle" demands that not only does the center of the universe lie everywhere, but also any fundamental observer must see the universe as isotropic and homogeneous. This means that, the space must be "maximally symmetric" [1]. By this consideration, one can directly obtain not only the metric for Einstein's static universe (ESU) but the non-static Friedmann-Robertson-Walker (FRW) metric as well [1,2] without using Einstein's equation $G_{i k}=8 \pi T_{i k}$ at all $(G=c=1)$. Let us invert it in the form

$$
T_{i k}=\frac{1}{8 \pi} G_{i k}
$$

The Einstein tensor $G_{i k}$, on the right hand side (RHS) of this equation, comprises only geometric quantities, the structure of space time $g_{i k}$. On the other hand, the left hand side (LHS) of the same equation contains much more tangible quantity, $T_{i k}$, the energy momentum tensor of the matter generating the space time structure $g_{i k}$ or $G_{i k}$. Thus any constraint imposed on $g_{i k}$ from symmetry or other considerations must take its toll on the admissible form of $T_{i k}$ as well. This means that constraints imposed on $G_{i k}$ must be reflected on the $T_{i k}$ of the fluid generating the $G_{i k}$. This latter physics aspect however may remain hidden in a purely symmetry driven mathematical derivation of the cosmological metric which does not invoke Einstein equation at all. And in this paper, we would like to explore the tacit constraints imposed on $T_{i k}$ by the requirements of the cosmological principle, "there is no preferred center", or "center lies everywhere". All the results obtained during this process will be also ratified by physical considerations as well.

In effect we shall be dealing with spherically symmetric static solutions of Einstein equation with the inclusion 
of a cosmological constant $\Lambda,[3,4]$. In recent times several authors have shown keen interest in this important problem [5-8]. However such studies mostly focus attention on isolated objects or stars where there (1) must be a pressure gradient, (2) in general, a density gradient, (3) a natural boundary $R=R_{b}$ where fluid pressure must vanish, $p\left(R_{b}\right)=0$ and (4) where a discontinuity in the fluid density $\rho$ might also occur. Further in such generalized studies, $\Lambda$ has not been considered as a fixed fundamental constant contrary to what was in Einstein's mind when it was first introduced.

In contrast, in the present study $\Lambda \equiv \Lambda_{\text {Einstein }}$ is a basic constant and there is no question of modifying its value according to the needs of a general fluid solution. And of course, there must not be any natural "boundary" at all for the universe so that Copernican principle remains valid. It may be recalled that the ancient Ionian philosopher "Aristarchos of Samos" had proposed the heliocentric theory much before Copernicus and hence we may also call this as "Aristarchian Principle". It will be found that both the metric for a star and ESU have a "coordinate singularity at $R=\alpha$, where $R$ is the luminosity distance and $\alpha$ is an appropriate integration constant. This singularity gets propagated in the hydrostatic balance equation as well. And in order to ensure that isotropic pressure gradient $p^{\prime}$ does not blow up at this singularity, relativistic stars are constructed so that $R_{b}>\alpha$. Here a prime denotes differentiation by R. Accordingly one can almost forget this singularity while studying static stars. But for the ESU one has to live with this singularity. And in this paper, we would study the behavior of physical quantities like pressure and acceleration despite the presence of this singularity. And the conclusion is that ESU has to be vacuous with $\Lambda=0$ in order to avoid such singularities anywhere.

At the very beginning, let us remind the difference between a fundamantal constant and a model parameter. Suppose we are studying a room temperature homogeneous static gas and we have obtained some expression for pressure $p$, temperature $T$ and particle number density $n$. But since $p=n k T$, in principle, we may obtain a numerical value of the Boltzmann constant $k$. Once we assume that $k$ is a fundamental constant and not just a model related parameter, the value of $k$ must be independent of time $t$ or position $r$ or any other variable. Thus, if we would be studying a gas in a situation where the gas may be inhomogeneous or may have bulk motion, we must be able to use the same numerical value of $k$ obtained in an ideal static homogeneous case. Similarly, here we would study the case of the supposed fundamental constant though it will be in the context of ESU. Therefore, we would expect $\Lambda=0$ for the dynamic FRW model too. It would be found that in order that the timelike worldlines of test particles always remain timelike and no trapped surface is formed, FRW model too has to be vacuous with $\Lambda=0$. Then the real universe must be something entirely different from the isotropic, homogeneous and continuous FRW/ESU models. Indeed galaxies and structures are found to be distributed in discrete, lumpy and inhomogeneous manner even at the largest scales. Nonetheless such matter distribution can still satisfy the "Copernican Principle" of no unique centre if it would form infinite hierarchal fractal pattern. At the beginning, however, we focus attention on the ESU.

\section{Formulation}

We start with the assumption of spherical symmetry and consider a general form a static metric [2]

$$
\mathrm{d} s^{2}=e^{v} \mathrm{~d} t^{2}-e^{\lambda} \mathrm{d} R^{2}-R^{2} \mathrm{~d} \Omega^{2}
$$

where $\mathrm{d} \Omega^{2}=\mathrm{d} \theta^{2}+\sin ^{2} \theta \mathrm{d} \varphi^{2}$ and $R$ is the area coordinate. In particular, in a static universe, the luminosity distance turns out to be exactly $R$ [2]

$$
\mathrm{d}_{L}=R
$$

We also assume the cosmic fluid to be perfect with

$$
T_{i k}=(\rho+p) u_{i} u_{k}-p g_{i k}
$$

where, $\rho$ is the fluid density, $p$ is the isotropic pressure, and $u^{i}$ is fluid 4-velocity. As is clear from Equation (1), at the beginning, we do not consider any cosmological constant $\Lambda$; and the ${ }_{0}^{0}$ component of the Einstein equation reads

$$
8 \pi T_{0}^{0}=8 \pi \rho=-e^{-\lambda}\left(\frac{1}{R^{2}}-\frac{\lambda^{\prime}}{R}\right)+\frac{1}{R^{2}}
$$

This can be integrated to yield

$$
e^{-\lambda}=1-\frac{\alpha(R)}{R}
$$

where

$$
\alpha(R)=\int_{0}^{R} 8 \pi \rho R^{2} \mathrm{~d} R
$$

Here the condition $\alpha(0)=0$ has been used to ensure that $e^{\lambda}$ is regular at $R=0$. Thus whether, it is the interior solution of a star or the static universe, general form of the metric is

$$
\mathrm{d} s^{2}=e^{v} \mathrm{~d} t^{2}-\frac{\mathrm{d} R^{2}}{1-\alpha / R}-R^{2} \mathrm{~d} \Omega^{2}
$$

Note, as of now, we need not necessarily interpret $\alpha(R)$ as related to the observed mass though what we have done looks like finding the interior of a relativistic star by working out the Schwarzschild interior solution. The important difference, however, is that while for a 
star there is a unique center and a boundary where the density may be discontinuous, for the universe, center is everywhere and there is no boundary, no exterior solution, and no density discontinuity. Further even for a constant density star, there must be a pressure gradient because there is a unique center. On the other hand, for an isotropic and homogeneous continuous universe, not only $p$ and $\rho$, but all physically meaningful quantities, all geometrical scalars must the same everywhere. Such requirements may demand severe restriction on the admissible equation of state (EOS) of the cosmic fluid. In contrast for a star, at best only $\rho$ can be uniform in which case there would be a discontinuity at its boundary $R=R_{b}$.

Now we introduce the condition that the fluid must be homogeneous: $\rho=$ uniform and thus both for the universe as well as a constant density star, one has

$$
\alpha=\frac{8 \pi}{3} \rho R^{3}
$$

and the metric assumes the form

$$
\mathrm{d} s^{2}=e^{v} \mathrm{~d} t^{2}-\frac{\mathrm{d} R^{2}}{1-(8 \pi / 3) \rho R^{2}}+R^{2} \mathrm{~d} \Omega^{2}
$$

If we introduce a parameter

$$
S=\sqrt{\frac{3}{8 \pi \rho}}
$$

Equation (10) would acquire the form

$$
\mathrm{d} s^{2}=e^{v} \mathrm{~d} t^{2}-\frac{\mathrm{d} R^{2}}{1-R^{2} / S^{2}}+R^{2} \mathrm{~d} \Omega^{2}
$$

It may be noted that Tolman [4] too obtained the static cosmological metric in a similar way by using the scalar $R$ as the radial variable. In the static case, $R$ is also a comoving coordinate for the interior solution because a given $R=$ fixed shell encloses a fixed number of baryons/particles.

Further, if we introduce a new coordinate

$$
r=R / S
$$

We can rewrite the above metric as

$$
\mathrm{d} s^{2}=e^{v} \mathrm{~d} t^{2}-S^{2}\left(\frac{\mathrm{d} r^{2}}{1-r^{2}}+r^{2} \mathrm{~d} \Omega^{2}\right)
$$

Note, the time dependent Robertson-Walker metric uses this $r$ as the (comoving) radial coordinate.

Hence, the spatial section of both the universe and the interior of a constant density star is that of a 3-sphere, a space of constant curvature, a fact noted by Weyl [3]. This becomes clearer if we express

$$
r=R / S=\sin \chi
$$

to rewrite Equation (14) as

$$
\mathrm{d} s^{2}=e^{v} \mathrm{~d} t^{2}-S^{2}\left(\mathrm{~d} \chi^{2}+\sin ^{2} \chi \mathrm{d} \Omega^{2}\right)
$$

There is clearly a singularity in one of the metric coefficients in Equations (12) and (14) at $R=S$ or $\alpha / R=1$ or $r=1$. On the other hand, metric (16) has a similar singularity at $\chi=0$. But one cannot make any a priori comment on the nature of such singularities without studying the behavior of relevant scalar quantities. Accordingly, we would try to study the behavior of scalars at appropriate regions instead of having any a priori debate/discussion on these singularities.

Let us suppose that somehow the density of the star would be reduced to zero everywhere. In such a case, the space time must become flat with a metric

$$
\mathrm{d} s^{2}=\mathrm{d} t^{2}-\mathrm{d} R^{2}-R^{2} \mathrm{~d} \Omega^{2}
$$

From the viewpoint of metric(8) it would immediately be apparent that one should then have $e^{v}=1$ and $\alpha / R=0$. But from the viewpoint of metric (14), it would appear that in such a case one would have $S=\infty$, $r=0$ and $R=S r \geq 0$. Why would we have $r=0$ in the latter case? This is so because the general form of a space time with constant spatial curvature is

$$
\mathrm{d} s^{2}=e^{v} \mathrm{~d} t^{2}-S^{2}\left(\frac{\mathrm{d} r^{2}}{1-K r^{2}}+r^{2} \mathrm{~d} \Omega^{2}\right)
$$

where $K=+1,0,-1$ corresponds to closed, flat and open space time respectively. And once we presume $K=+1$ and adopt metric (14), we presume $\rho>0$ and then it becomes difficult to have a smooth transition to a $\rho \rightarrow 0$ case. Hence, if one would try to arrive at a flat space $(K=0)$ by imagining that it is due to a closed space of infinite radius, one would land up with the condition $r=0$. In fact, if in Equation (4), had we taken,

$$
T_{0}^{0}=K \rho
$$

instead of $\rho$, we would indeed have obtained Equation (18) directly in lieu of Equation (14). Then we would have found that the condition for flatness is $K=0$. But when we do take $T_{0}^{0}=\rho$ and implicitly assume $\rho>0$, we presume $K=1$ and exclude the possibility that one might have $K=0$ too. In such a case, the condition for flatness would appear as $r=0$ as mentioned above. Note, the fact that the comoving coordinate $r=0$ signifies that there is no baryon/particle at all; i.e., it is all vacuum.

\section{Cosmological Constant}

It is well known that in order to obtain a static universe which would be closed and finite radius, Einstein modified Equation (1) into

$$
G_{i k}+\Lambda g_{i k}=8 \pi T_{i k}
$$


apparently implying that either $\Lambda$ is a fundamental constant like $8 \pi G / c^{4}$ or a basic scalar, like the Ricci scalar $R$ appearing within $G_{i k}$. It is also well known that, from a purely mathematical view point, one can incorporate the effect of $\Lambda$, by replacing [7]

$$
\rho \rightarrow \rho_{e}=\rho+\frac{\Lambda}{8 \pi}
$$

and

$$
p \rightarrow p_{e}=p-\frac{\Lambda}{8 \pi}
$$

It should be borne in mind that such a mathematical clubbing however does not really make $\Lambda$ a new form of matter. This is so because while real matter represented by $\rho$ generates global negative self-gravitational energy, pure vacuum represented by $\Lambda$ is not associated with any negative self-gravitational energy.

So, as far as mathematics is concerned, instead of Equation (7), one now obtains

$$
e^{-\lambda}=1-\frac{\alpha_{e}(R)}{R}
$$

where

$$
\alpha_{e}=\int_{0}^{R} 8 \pi \rho_{e} R^{2} \mathrm{~d} R
$$

One also finds

$$
\mathrm{d} s^{2}=e^{v} \mathrm{~d} t^{2}-\frac{\mathrm{d} R^{2}}{1-\alpha_{e} / R}-R^{2} \mathrm{~d} \Omega^{2}
$$

And for a constant density case, one has

$$
\mathrm{d} s^{2}=e^{v} \mathrm{~d} t^{2}-\frac{\mathrm{d} R^{2}}{1-(8 \pi / 3) \rho_{e} R^{2}}+R^{2} \mathrm{~d} \Omega^{2}
$$

and

$$
S=\sqrt{\frac{3}{8 \pi \rho_{e}}}
$$

Equations (12)-(16) remain unaltered in the presence of $\Lambda$. Thus now, if the space time has to be flat, in addition to $e^{v}=1$, one must have

$$
\rho_{e} \rightarrow 0
$$

This shows that if the original definition of "vacuum" is $\rho=0$, as if, in the presence of a $\Lambda$, it gets modified to $\rho_{e}=0$.

\section{Acceleration Scalar and Singularity}

The fact that for a spherically symmetric static system, one should have $\alpha / R<1$ as has already been investigated [8-10]. The basic reason for this is not difficult to see. In general, static or non-static, for spherically sym- metric space time occurrence of $\alpha(r, t) / R>$ corresponds to a formation of a "trapped surface" and the condition $\alpha(r, t) / R=1$ marks the formation of an "apparent horizon". Though for a non-static system, it is conjectured to be possible to have trapped surfaces or horizons, for a static system they are not allowed. This is so because once a trapped surface would be there, stellar matter would be inexorably pulled towards the central point of symmetry and thus matter would soon end up in a point singularity rather than as an extended static object. While this is definitely not allowed for a static star, this problem would be much more severe for cosmology because "center of symmetry lies everywhere".

Following the case of a Schwarzschild black hole space time, generally, it is believed that this $R=\alpha$ singularity is a mere coordinate one even in the presence of matter. But as we would see below, the coordinate independent scalar acceleration of the fluid would blow up unless severe constraints are imposed on the fluid EOS.

It may be recalled that Einstein was very much concerned about this $R=\alpha$ singularity and constructed a static model of a fluid where test particles are moving in randomly oriented circular orbits under their own gravitational field. While the radial stress of the fluid is zero, tangential stresses are finite. This configuaration is known as "Einstein Cluster" and Einstein showed that, the speed of the orbiting particles would be equal to the speed of light $c$ at $R=\alpha$ [11]. Thus, he pleaded that there cannot be any $R=\alpha$ or $R<\alpha$ situation. Later it turned out that Einstein's cluster indeed corresponded to some well defined interior Schwarzschild solution studied by Florides [12,13]. And now Bohmer \& Lobo [14] have shown that Einstein's intuition was correct, and the $R=\alpha$ singularity is indeed a curvature singularity. This however does not at all mean that all $R=\alpha$ singularities are curvature singularities. In fact, in the present static case of isotropic pressure, it would be found that they should be regions with zero curvature singularity to avoid an acceleration or pressure gradient singularities.

For any static spherically symmetric fluid, one can easily find the acceleration [8]

$$
a^{i}=u^{k} \nabla_{k} u^{i}
$$

In spherical symmetry, only one component of acceleration survives

$$
a^{R}=\frac{e^{-\lambda} v^{\prime}}{2}
$$

How to evaluate this $a^{R}$ ? Again, for the sake of easy understanding, we first do not consider any $\Lambda$ and write down the $\begin{array}{ll}R \\ R\end{array}$ component of Einstein Equation (1), 


$$
8 \pi T_{R}^{R}=-8 \pi p=-e^{-\lambda}\left(\frac{v^{\prime}}{R}+\frac{1}{R^{2}}\right)+\frac{1}{R^{2}}
$$

In view of Equation (30), let us rewrite this equation as

$$
-\frac{e^{-\lambda}}{R} v^{\prime}=\frac{\alpha+8 \pi p R^{3}}{R^{3}}
$$

or

$$
a^{R}=-\frac{\alpha+8 \pi R^{3} p}{2 R^{2}}
$$

Since there is only one component of $a^{i}$, the scalar acceleration becomes

$$
a=\sqrt{-a^{i} a_{i}}=a^{R} \sqrt{\left|g_{R R}\right|}=\frac{e^{-\lambda / 2}\left|v^{\prime}\right|}{2}
$$

Using Equation (32), we obtain

$$
a=\frac{\alpha+8 \pi R^{3} p}{2 R^{2} \sqrt{1-\alpha / R}}
$$

If $\Lambda$ would be included, then the acceleration scalar would be given by

$$
a=\frac{\alpha_{e}+8 \pi R^{3} p_{e}}{2 R^{2} \sqrt{1-\alpha_{e} / R}}
$$

It is now clear that if a static fluid would tread upon the $R=\alpha_{e}$ singularity, its acceleration could be infinite and it cannot be at rest. And this is the physical reason that for a static fluid one must have $R>\alpha_{e}$ everywhere. And in case, the manifold would cover $R=\alpha_{e}$, one must satisfy

$$
\alpha_{e}+8 \pi R^{3} p_{e}=0 ; \quad R=\alpha_{e}
$$

in an attempt to keep $a$ regular. Using $R=\alpha_{e}$ in the foregoing equation, we have

$$
\alpha_{e}\left(1+8 \pi \alpha_{e}^{2} p_{e}\right)=0
$$

i.e., one must have either

$$
\alpha_{e}=0
$$

or,

$$
p_{e}\left(R=\alpha_{e}\right)=-\frac{1}{8 \pi \alpha_{e}^{2}}
$$

or both of the above two conditions. If we assume that the minimum value of $p$ is zero and it cannot be negative, we will have $p_{e}=-\Lambda / 8 \pi$. Then Equation (40) would yield

$$
R=S=\sqrt{\frac{1}{\Lambda}}
$$

But one is still not sure whether the additional condi- tion such as Equation (39) is needed to really ensure that $a$ is indeed finite at $R=\alpha_{e}$. For a fluid with $\rho_{e}>0$, the safest way to avoid the $R=\alpha_{e}$ singularity will simply be to ensure that $R>\alpha_{e}$. The fluid can do so by choosing an appropriate density profile and by ensuring that its outer boundary

$$
R_{b}>\alpha_{e}
$$

For a constant density star or the ESU, we have

$$
a=\kappa \frac{R\left(\rho_{e}+3 p_{e}\right)}{\sqrt{1-2 \kappa \rho_{e} R^{2}}}
$$

where $\kappa=4 \pi / 3$. In terms of $r$, we obtain

$$
a=\kappa S \frac{r\left(\rho_{e}+3 p_{e}\right)}{\sqrt{1-r^{2}}}
$$

It is clear that the sufficient condition for avoiding the $R=\alpha_{e}$ singularity in this case is

$$
R_{b}>\alpha ; \quad R<S
$$

In terms of density, this means

$$
\rho_{e}>\frac{3}{8 \pi R_{b}^{2}}
$$

Thus, if one would imagine a region with $\rho=0$, i.e., $\rho_{e}=\Lambda / 8 \pi$, one should restrict the radius of this vacuum as

$$
R_{b}<\sqrt{\frac{3}{\Lambda}}
$$

And in case, one would have $\rho=\Lambda / 4 \pi$, then the above restriction would become

$$
R_{b}<\sqrt{\frac{1}{\Lambda}}
$$

and which cannot be satisfied. Thus for a constant density star with $R_{b}=\sqrt{1 / \Lambda}$, one must have $\rho>\Lambda / 4 \pi$. And in case this condition would be violated, one must critically analyze the additional constraint on the EOS which would prevent $p_{e^{\prime}}$ from blowing up at $R=\alpha_{e}$. It may be seen that the $\rho=\Lambda / 4 \pi \quad$ EOS corresponds to

$$
\rho_{e}+3 p_{e}=0
$$

if $p=0$.

\section{TOV Equation}

Recall that local energy momentum conservation equation $T_{i ; k}^{k}=0$ immediately leads to [2]

$$
v^{\prime}=\frac{-2 p^{\prime}}{p+\rho}
$$

Further if we again consider the ${ }_{R}^{R}$ component of 
Einstein Equation and combine it with Equation (50), we will obtain the TOV equation for hydrostatic balance for any self-gravitating static fluid:

$$
p^{\prime}=-\frac{(\rho+p)\left(\alpha+8 \pi R^{3} p\right)}{2 R^{2}(1-\alpha / R)}
$$

Since the effect of $\Lambda$ gets included if one replaces $p$ and $\rho$ by their "effective" values[7]

$$
p^{\prime}=-\frac{\left(\rho_{e}+p_{e}\right)\left(\alpha_{e}+8 \pi R^{3} p_{e}\right)}{2 R^{2}\left(1-\alpha_{e} / R\right)}
$$

Further since $p^{\prime}=p_{e}^{\prime}$, in a complete symmetric manner, we rewrite the above equation as

$$
p_{e}^{\prime}=-\frac{\left(\rho_{e}+p_{e}\right)\left(\alpha_{e}+8 \pi R^{3} p_{e}\right)}{2 R^{2}\left(1-\alpha_{e} / R\right)}
$$

This equation strongly suggests that if the definition of a "dust" in the absence of $\Lambda$ is $p=0$, as if, in the presence of $\Lambda$, dust EOS should be $p_{e}=0$. Similarly, if the original EOS of "vacuum" is $\rho=0$, in the presence of $\Lambda$, the vacuum EOS is $\rho_{e}=0$, a hint we have already found.

Note, even now, there is no need to interpret $\alpha$ in terms of any exterior boundary condition, and thus TOV equation is valid in any spherically symmetric static GR problem including ESU. By using Equation (36), it is interesting to rewrite the TOV Equation in terms of the acceleration scalar

$$
p_{e}^{\prime}=-\frac{a\left(\rho_{e}+p_{e}\right)}{\sqrt{1-\alpha_{e} / R}}
$$

Clearly, there is a singularity in the denominator of TOV Equation at $R=\alpha_{e}$. If we write

$$
x=\sqrt{1-\alpha_{e} / R}
$$

It is seen that while the singularity in acceleration $a \sim x^{-1}$, for the pressure gradient it is much stronger $p^{\prime} \sim x^{-2}$. And if the fluid would cover the $R=\alpha_{e}$ singularity, regularity of $a$ may not be sufficient to ensure regularity of $p^{\prime}$. In fact Equation (54) suggests that one might require the additional constraint

$$
\rho_{e}+p_{e}=0 ; \quad R=S
$$

to tame the $p^{\prime}$ singularity at $R=S$.

Now let us use the condition $\rho_{e}=$ uniform which is equally valid for the interior solution of a constant density star as well as the ESU:

$$
p^{\prime}=-\kappa R \frac{\left(\rho_{e}+p_{e}\right)\left(\rho_{e}+3 p_{e}\right)}{1-2 \kappa \rho_{e} R^{2}}
$$

In terms of the normalized coordinate $r$, one finds

$$
p^{\prime}=-\kappa S \frac{r\left(\rho_{e}+p_{e}\right)\left(\rho_{e}+3 p_{e}\right)}{1-r^{2}}
$$

And in term of $a$, we have

$$
p^{\prime}=-\frac{a\left(\rho_{e}+p_{e}\right)}{\sqrt{1-2 \kappa \rho_{e} R^{2}}}
$$

and

$$
p^{\prime}=-\frac{a\left(\rho_{e}+p_{e}\right)}{\sqrt{1-r^{2}}}
$$

\section{Constant Density Star}

For the interior solution of a constant density relativistic star one, one finds [7]

$$
e^{v}=\left(\frac{p^{c}+\rho}{p+\rho}\right)^{2}
$$

where $p_{c}$ is the central pressure. This relation is interesting because it does not involve any exterior bounday condition. Also note that since

$$
p+\rho=p_{e}+\rho_{e}
$$

we can rewrite Equation (61) as

$$
e^{v}=\left(\frac{p_{e}^{c}+\rho_{e}}{p_{e}+\rho_{e}}\right)^{2}
$$

Since for a star, there must be a pressure gradient, $p^{c} \neq p(R)$, and one really cannot reduce $e^{v}(R)$ to a constant value independent of $R$. But if, from the mathematical viewpoint, one would still demand that it should be possible to set up a time orthogonal Gaussian coordinate system where $d t^{2}=d \tau^{2}$, one would unknowingly kill the pressure gradient and set $p^{c}=p$ in Equation (61). In the absence of $\Lambda$, for a static configuration, $\rho=0$ if $p=0$, and the star would vanish under the assumption of $e^{v}=1$ !

When $\Lambda$ is present, the expression for pressure for a constant density star is [7]

$$
p(R)=\rho \frac{\Lambda / 4 \pi-1+C \cos \chi}{3-\cos \chi}
$$

where

$$
C=\frac{3 p^{c}+\rho-\Lambda / 4 \pi}{p^{c}+\rho}=\frac{3 p_{e}^{c}+\rho_{e}}{p_{e}^{c}+\rho_{e}}
$$

Now it transpires that even when $p=0$ to honor $e^{v}=1$, there would still be a finite density $\rho=\Lambda / 4 \pi$. But one attains this finite density at a huge cost because it now turns out that the boundary of the star merges with the coordinate singularity $R_{b}=S$ where the metric 
behaves badly. For a real star, this singularity can always be avoided by allowing $e^{v}>1$ which in turn means by having $\rho>\Lambda / 4 \pi$. Thus a "world time" cannot be introduced for a general relativistic star having a finite density.

\section{Verification from TOV Equation}

Although the case of constant density stars in the presence of cosmological constants has been studied in great detail, to our knowledge, nobody actually verified the validity of the solutions in case one would tread on the coordinate singularity at $R=S$. Since the denominator of Equation (53) becomes zero at $R=S$, in order that $p^{\prime}$ does not blow up there, one must have either

$$
\rho_{e}+p_{e}=0 ; \quad R=S
$$

or

$$
\rho_{e}+3 p_{e}=0 ; \quad R=S
$$

or both

$$
\rho_{e}+p_{e}=\rho_{e}+3 p_{e}=0 ; \quad R=S
$$

If the last constraint would be satisfied, one would immediately obtain $\rho_{e}=p_{e}=0$. However, it is possible that Equation (68) is not needed and only one of the less rigourous conditions (66) or (67) is satisfied. In particular, to avoid occurrence of negative pressure, let us assume that only Equation (67) is satisfied at $R=S$. Since $p^{\prime}$ attains a $0 / 0$ form at $R=S$, let us study the nature of $p^{\prime}$ at this singularity by using L' Hospital rule. For this, let us first write

$$
p_{e^{\prime}}=\frac{f(R)}{g(R)}
$$

where

$$
f(R)=\kappa R\left(\rho_{e}+p_{e}\right)\left(\rho_{e}+3 p_{e}\right)
$$

and

$$
g(R)=2 \kappa \rho_{e} R^{2}-1
$$

Since $\rho^{\prime}=0$, we find that

$$
\begin{aligned}
f^{\prime} & =\kappa\left(\rho_{e}+p_{e}\right)\left(\rho_{e}+3 p_{e}\right) \\
& +\kappa R p_{e}^{\prime}\left[3\left(\rho_{e}+p_{e}\right)\left(\rho_{e}+3 p_{e}\right)\right]
\end{aligned}
$$

Also, since, we have already considered $\rho_{e}+3 p_{e}=0$, we obtain a reduced expression for

$$
f^{\prime}=3 \kappa R p_{e^{\prime}}\left(\rho_{e}+p_{e}\right)
$$

On the other hand,

$$
g^{\prime}=4 \kappa \rho_{e} R
$$

So that

$$
f^{\prime} / g^{\prime}=3 p_{e^{\prime}} \frac{\rho_{e}+p_{e}}{4 \rho_{e}}
$$

And at the singularity, $R=S$, by l' Hospital rule, we obtain

$$
p_{e^{\prime}}=\lim _{R \rightarrow S} \frac{f^{\prime}}{g^{\prime}}
$$

From Equations (75) and (76) we obtain the required condition:

$$
3\left(\rho_{e}+p_{e}\right)=4 \rho_{e}
$$

i.e.,

$$
\rho_{e}(R=S)=3 p_{e}(R=S)
$$

which looks like the EOS of incoherent radiation! By combining Equations (67) and (78), it becomes clear that in order that $p^{\prime}$ does not blow up at the "coordinate singularity" at $R=S$, the constant density star must have

$$
\rho_{e}(R=S)=p_{e}(R=S)=0
$$

Thus instead of $p=0$ we would obtain $p_{e}=0$ at $R=S$. This means that if indeed $\Lambda>0$, the solution must avoid $R=S$ singularity. More importantly, since $\rho_{e}=$ constant, we find that, if the solution would indeed extend upto $R=S$, we must have $\rho_{e}=0$. Thus all finite density stars must avoid the $\alpha_{e} / R=1$ or $r=1$ singularity.

\section{Static Universe}

In cosmological case, there must be a universal time which would be the proper time of all fundamental observers, i.e., $d t^{2}=d \tau^{2}$. This demands that one must be able to set $e^{v}=1$ so that, the Equation (14) would become the ESU metric

$$
\mathrm{d} s^{2}=\mathrm{d} t^{2}-S^{2}\left(\frac{\mathrm{d} r^{2}}{1-r^{2}}+r^{2} \mathrm{~d} \Omega^{2}\right)
$$

Note that the value of $S$ for ESU is still given by Equation (27) and thus one is justified in deducing ESU metric indeed by setting $e^{v}=1$. Since the Einstein equation tells that the metric is essentially determined by $T_{i k}$, such an important change of setting $e^{v}=1$ on the LHS of this equation must be endorsed by the RHS, i.e., by the admissible forms of fluid EOS. What are those conditions? To explore them, we note from Equations (50) and (51) that the most general form of static, spherically symmetric Einstein Equation yields

$$
v^{\prime}=-\frac{\alpha_{e}+8 \pi R^{3} p_{e}}{R^{2}\left(1-\alpha_{e} / R\right)}
$$

In constant density case, this condition becomes

$$
v^{\prime}=-2 \kappa \frac{R\left(\rho_{e}+3 p_{e}\right)}{1-2 \kappa \rho_{e} R^{2}}
$$


And in term of $r$, this condition becomes

$$
v^{\prime}=-2 \kappa S \frac{r\left(\rho_{e}+3 p_{e}\right)}{1-r^{2}}
$$

In order that $e^{v}=1$, one must have $v^{\prime}=0$, and from the foregoing equation, it is immediately clear that then we must have atleast

$$
\rho_{e}+3 p_{e}=0
$$

In any case, we must have $p_{e^{\prime}}=0$ everywhere including $R=S$ or $r=1$. Then if we follow the L' Hospital treatment of the previous section, we would find, we must have

$$
\rho_{e}=0
$$

everywhere because $\rho_{e}=$ constant . Although, we have already obtained this important result, it would be interesting to obtain this result from somewhat different routes.

First consider Equation (59) and write

$$
f(R)=a\left(\rho_{e}+p_{e}\right)
$$

and

$$
g(R)=-\sqrt{1-2 \kappa \rho_{e} R^{2}}
$$

so that

$$
f^{\prime}=p_{e^{\prime}} a
$$

and

$$
g^{\prime}=2 \kappa \rho_{e}\left(1-2 \kappa \rho_{e} R^{2}\right)^{-1 / 2}
$$

Now applying L'Hospital rule at $R=S$, we find

$$
p^{\prime}=p^{\prime} \frac{a \sqrt{1-2 \kappa \rho_{e} R^{2}}}{2 \kappa \rho_{e} R}
$$

Since the $\lim x / x=1$ irrespective of whether $x \rightarrow 0$ or $x \rightarrow \infty$ or $x=$ finite, we can cancel $p^{\prime}$ from both sides of the foregoing equation. Then, using Equation (43) in the above Equation, we find

$$
\frac{\rho_{e}+3 p_{e}}{2 \rho_{e}}=1
$$

This again implies

$$
\rho_{e}=3 p_{e}
$$

In conjunction with (68), this will lead to $\rho_{e}=p_{e}=0$. It may be also of some interest to extend this study by directly considering the radial variable as $r$ rather than $R$. Note that

$$
p_{e^{\prime}}=\frac{1}{S} \mathrm{~d} p_{e} / \mathrm{d} r
$$

Now if we denote differentiation by $r$ with a prime, we rewrite Equation (60) as

$$
p^{\prime}=-\frac{S a\left(\rho_{e}+p_{e}\right)}{\sqrt{1-r^{2}}}
$$

so that

$$
f(r)=S a\left(\rho_{e}+p_{e}\right)
$$

and

$$
g(r)=-\sqrt{1-r^{2}}
$$

Then we have

$$
f^{\prime}=S a p^{\prime}
$$

and

$$
g^{\prime}=\frac{r}{\sqrt{1-r^{2}}}
$$

Again applying L'Hospital rule for the limit of $p^{\prime}$ at $R=S$, we find

$$
\frac{S a \sqrt{1-r^{2}}}{r}=1
$$

Inserting the expression for $a$ from Equation (44), we obtain the interesting relation

$$
\kappa S^{2}\left(\rho_{e}+3 p_{e}\right)=1
$$

or

$$
S=\sqrt{\frac{3}{4 \pi\left(\rho_{e}+3 p_{e}\right)}}
$$

Comparison of Equations (27) and (101) would again convey one of the hidden messages for the ESU as well as for a constant density star which is attemting to suppress its pressure gradient, namely $3 p_{e}=\rho_{e}$. The sum and substance of the entire exercise is that for the ESU, in oder that $p_{e^{\prime}}$ is indeed zero everywhere, one must have

$$
\rho_{e}=3 p_{e}=0
$$

But unlike the constant density stellar case now we cannot escape confronting this singularity by demanding that $\rho>\Lambda / 4 \pi$. And hence we must accept the fact that for the ESU fluid $\rho_{e}=p_{e}=0$ everywhere. This means that

$$
\rho+\Lambda / 8 \pi=0 ; \quad \rho=-\Lambda / 8 \pi
$$

atleast for the ESU. The weak energy condition demands that $\rho \geq 0$ and thus we find that $\Lambda=0$ !

\section{Einstein's Solution}

If one would directly use Equation (80) into Einstein Equation (20), one would be led to[4] 


$$
4 \pi(\rho+3 p)=\Lambda
$$

and

$$
4 \pi(\rho+p)=\frac{1}{S^{2}}
$$

Einstein chose, $p=0$ EOS which led to

$$
1 / S^{2}=\Lambda ; \quad S=\sqrt{\frac{1}{\Lambda}}
$$

Note that Equations (104) and (105) may be rewritten as

$$
4 \pi\left(\rho_{e}+3 p_{e}\right)=0
$$

and

$$
4 \pi\left(\rho_{e}+p_{e}\right)=\frac{1}{S^{2}}
$$

But, if we recall, Equation (92), actually, $\rho_{e}+p_{e}=0$ so that $S=\infty$. Further Equations (100)-(101) showed that

$$
4 \pi\left(\rho_{e}+3 p_{e}\right)=\frac{3}{S^{2}}
$$

And a comparison of Equations (108) and (109) again shows that, $S=\infty$ and $\rho_{e}=0$. As mentioned before, for weak energy condition, this would mean $\rho=\Lambda=0$. Note that it is indeed possible to have a situation where $\rho_{\text {rest }} / e \gg 1$, where $e$ is the proper internal energy density. In such a case, one might approximately write $p \approx 0$. But this does not mean that pressure is strictly zero. A strict $p=0$ EOS is possible only when $\rho=0$. In such a case, Equation (104) would again yield $\Lambda=0$.

However, if we would ignore such physical and mathematical regularity or self-consistency considerations, Einstein's solution (106) would apparently suggest $\Lambda=4 \pi G \rho / c^{2}>0$.

Further, if one would assume that (1) ESU correctly describes the real universe and (2) the true mean density of the real universe in its totality (about which we may never have an absolute knowledge) is equal to the mean density of the patch of the observed universe, i.e.,

$$
\rho_{\text {patch }}=\rho_{\text {true }}=\rho \sim 10^{-31} \mathrm{~g} \mathrm{~cm}^{-3}
$$

one would obtain[2]

$$
\Lambda \sim 10^{-58} \mathrm{~cm}^{2}
$$

Now ponder over the fact that until the advent of big optical telescopes in 1920 or so, for hundreds of years, most of the astronomers thought that total universe was nothing but our Milkyway galaxy. And if would have been possible to measure the mean density of the Milkyway, one might have concluded that

$$
\rho_{\text {patch }}=\rho_{\text {true }}=\rho \sim 10^{-13} \mathrm{~g} \mathrm{~cm}^{-3}
$$

and accordingly

$$
\Lambda \sim 10^{-40} \mathrm{~cm}^{2} \text { before } 1920
$$

As late as 1970, we hardly had any idea that the galaxy clusters and superclusters are actually distributed as "filaments" and "walls" around huge voids whose dimensions could be as large as $\sim 280 \mathrm{Mpc}$ [15]. The recent Sloan Digital Sky Survey has revealed structures of dimension $\sim 500 \mathrm{Mpc}$ [16]. Thus, we cannot rule out the possibility that eventually, it might be found that the entire patch of presently observed universe also lies on the wall of a larger void. If the mean density of observed universe would be revised by future observations, would we again revise the value of the fundamental constant $\Lambda$ ?

At the cost of sounding repetitive, let us again raise the old question: if $\Lambda$ would indeed be due to some quantum mechanical effect, why the value of $\Lambda$ obtained under the assumption of $\rho_{\text {patch }}=\rho_{\text {true }}$ falls short of the theoretical value by an approximate factor of $10^{120}$ ? And if the various field modes would cancel one another to generate a small $\Lambda$, why do they not cancel exactly to result $\Lambda=0$ ? In fact there are some theoretical estimates by which $\Lambda=0$ [17].

\section{Summary So Far}

Both a spherically symmetric static star and the ESU result from the same spherically symmetrical form of Einstein equations. If ${ }_{R}^{R}$ component of the Einstein equation would be studied, one would obtain, the expression for acceleration scalar and condition for hydrostatic balance in both the cases. In fact, Tolman [4] did obtain one of the constraints to be followed by the ESU fluid by demanding that the pressure gradient $p^{\prime}$ must vanish for the ESU: $\rho_{e}+3 p_{e}=0$. We found that the singularity appearing in the $(R, R)$ component of the metric tensor in all spherically symmetric static configurations do propagate in the expressions for acceleration scalar $a$ and pressure gradient $p^{\prime}$. While, for the former, the nature of singularity is $a \sim x^{-1}$, for the latter it is much stronger $p^{\prime} \sim x^{-2}$. In fact, the requirement $\rho_{e}+3 p_{e}=0$ is the basic condition for ensuring that the $a$ does not blow up at $x=0$ or $r=1$. This basic condition however need not be the sufficient condition for ensuring that $a$ is finite at $x=0$. Further, since $p^{\prime}$ tends to blow up much faster at $x=0$, one must require an additional condition to ensure the regularity of $p^{\prime}$. We found that the latter requirement is $\rho_{e}=3 p_{e}$. This means that if the constant density static star would have $R_{b}=S$, one must have $\rho_{e}=0$. But one can always avoid this singularity by ensuring that $\rho>\Lambda / 4 \pi$. However, previous detail studies of perfect fluid spheres were primarily in- 
tended for isolated systems and not for the universe, and to our knowledge, nobody tried to study the remedy for either the $\sim x^{-1}$ or $\sim x^{-2}$ singularities at $x=0$.

Incidentally, it has been found that even for a supposed constant density star with $R_{b}<\alpha$, the "constant density" is nothing but $\rho=0 \quad$ [18].

\section{Buchdahl Inequality}

It is well known that any spherically symmetric static configuration, homogeneous or inhomogeneous, supported by isotropic pressure, satisfies a constraint much stronger than the $R_{b}>\alpha$ or $\alpha<R_{b}$ constraint. In the absence of a $\Lambda$, this is known as Buchdahl inequality [19]:

$$
\frac{\alpha}{R_{b}}<\frac{8}{9}
$$

If one would have $\alpha=(8 / 9) R_{b}$, central pressure would blow up (assuming $\rho>0$ ). For a uniform density star this borderline would correspond to

$$
r=8 / 9
$$

But suppose there is a solution which appears to violate this constraint, i.e., $r \geq 8 / 9$. Then how to ensure that central pressure is still finite? The only solution out of this dilemma would be to set $\alpha=0$ or $\rho=0$. From this more stringent condition, it should be clear why Einstein's original static universe must have $\rho=0$.

Does the fate of the ESU improve after the incorporation of a positive $\Lambda$ ? From Equation (3.35) of ref.[7], it turns out that now the modified Buchdhal constraint is

$$
\frac{\alpha_{e}}{R}<\frac{2}{9}\left(4-\frac{\Lambda}{4 \pi \rho}\right)
$$

Thus with a positive $\Lambda$, this constraint gets even tighter. With $\rho=\Lambda / 4 \pi$, one obtains

$$
\frac{\alpha_{e}}{R}<\frac{2}{3}
$$

In view of Equations (9), (11) and (33), this means that for a constant density star, one must have

$$
r<\sqrt{2 / 3}
$$

to ensure that pressure does not blow up at the center $r=0$ ! For the ESU, this would mean blowing up of pressure everywhere because "center would lie everywhere". And the only solution to get rid of this problem is is accept the fact that $\alpha_{e}=0$ for ESU.

\subsection{Mass Function}

Since for a fluid in an asympotictially flat space time, one can define a "mass function" in terms of which acceleration scalar

$$
a=\frac{M+4 \pi R^{3} p}{R^{2} \sqrt{1-2 M / R}}
$$

we can now identify $\alpha(R)$ in Equation (36) as twice the quasilocal mass-energy of the fluid

$$
\alpha(R)=2 M(R)
$$

In the presence of a $\Lambda$, the quasilocal mass will be $M_{e}=\alpha_{e} / 2$.

In general relativity, the gravitational mass of a stationary system is[3]

$$
M=\int\left(T_{0}^{0}+t_{0}^{0}\right) \sqrt{-g} \mathrm{~d}^{3} x
$$

where $t_{i}^{k}$ is energy momentum pseudo tensor and $g$ is the determinant of the metric tensor. One can work out $t_{0}^{0}$ from the metric $g_{i k}$ of the universe. Probably starting from Rosen [20], many authors have worked out the mass energy of a closed universe and all of them have concluded that for a closed universe $M=0$ [21-25]. This too would suggest that, in the absence of $\Lambda$, $\rho=0$, and, in general $\rho_{e}=0$. For the ESU, it is possible to confirm this result irrespective of the value of $t_{0}^{0}$. In all coordinate systems, for a static fluid, one obtains [2-4]

$$
\begin{aligned}
M_{e} & =\int(\rho+3 p-\Lambda / 4 \pi) \sqrt{-g} \mathrm{~d}^{3} x \\
& =\int\left(\rho_{e}+3 p_{e}\right) \sqrt{-g} \mathrm{~d}^{3} x
\end{aligned}
$$

In view of Equation (84) we thus directly obtain

$$
M_{e}=0
$$

Further, from Equation (24), we obtain $\rho_{e}=0$

\subsection{Poisson Equation}

It is known that the RHS of Poission equation indicates the source of gravity. For a spherically symmetric static fluid the Poission's equation is [26,27]

$$
\begin{aligned}
\nabla^{2} \sqrt{g_{00}} & =4 \pi \sqrt{g_{00}}(\rho+3 p-\Lambda / 4 \pi) \\
& =4 \pi \sqrt{g_{00}}\left(\rho_{e}+3 p_{e}\right)
\end{aligned}
$$

This shows that the source density of gravity is

$$
\rho_{g}=\sqrt{g_{00}}\left(\rho_{e}+3 p_{e}\right)
$$

And when $g_{00}=1$, one will obtain

$$
\rho_{g}=\left(\rho_{e}+3 p_{e}\right)
$$

In either case, the RHS of Equation (124) is zero when $\rho_{e}+3 p_{e}=0$, which is the case for both a constant density star (if it would be extended up to $R=S$ ) or the 
ESU. In all such cases, one must necessarily have $g_{00}=e^{v}=$ constant . And since source of gravity is zero, the space time must be flat. And a space time is flat when $\rho_{e}=0$, as obtained by us. Alternatively, if one would first focus attention on the LHS of Equation (124), it seems that if one would set $e^{v}=$ constant, the source of gravity will vanish, again in which case, one should have $\rho_{e}=0$.

\section{A Simple Reason}

Suppose one chooses to ignore most of the previous proofs that $\rho_{e}=0$ for ESU in order to avoid any physically singular behavior. Even then, one can arrive at the same conclusion by simply demanding that all physical quantities must be position independent in accordance with the inherent assumption in the following manner:

For a constant density case, we may rearrange the TOV equation(58) as

$$
\frac{-p^{\prime}}{\left(\rho_{e}+p_{e}\right)\left(\rho_{e}+3 p_{e}\right)}=\frac{\kappa S r}{1-r^{2}}
$$

We may also rewrite the acceleration Equation (44) as

$$
\frac{a}{\rho_{e}+3 p_{e}}=\frac{\kappa S r}{\sqrt{1-r^{2}}}
$$

The RHS of Equations (127) and (128) obviously depends on $r$ if $r$ would indeed be a free parameter. For a constant density star, the LHS of the same equations too must depend on $r$. And they do depend on $r$ because $p_{e}=p_{e}(r)$ even though $\rho_{e}=$ constant . But suppose we would like to freeze the $r$ dependence of $p$ to make $e^{v}=1$. Then the value of $r$ on the RHS of Equations (127) and (128) too must be frozen. And since $R=S r \geq 0$ during the freezing process, the only solution here would be to adopt

$$
S=\infty ; \quad r=0 ; \quad R \geq 0
$$

We have already discussed that occurrence of Equation (129) actually signifies that the spatial section is flat: $K=0$. This would have been more transparent had we taken

$$
T_{1}^{1}=T_{2}^{2}=T_{3}^{3}=-K p
$$

alongwith Equation (19), i.e., had we written

$$
T_{i k}=K\left[(\rho+p) u_{i} u_{k}+p g_{i k}\right]
$$

instead of Equation (4). Further had we also taken the cosmological constant as $K \Lambda$ instead of $\Lambda$, Equations (127) and (128) would have appeared as

$$
\frac{-p^{\prime}}{\left(\rho_{e}+p_{e}\right)\left(\rho_{e}+3 p_{e}\right)}=\frac{K \kappa S r}{1-K r^{2}}
$$

and

$$
\frac{a}{\rho_{e}+3 p_{e}}=\frac{K \kappa S r}{\sqrt{1-K r^{2}}}
$$

respectively. In such a case, it would have been seen immediately that the requirement that the RHS of Equations (132) and (133) are independent of $r$, one must have is $K=0$. And since we have already set $e^{v}=1$, it means, the space time must be flat to ensure that the RHS of Equations (127) and (128) or (132) and (133) are indeed independent of $r$. Hence one must have $K \rho_{e}=0$ in such a case. So will be the case for ESU. This means that in order that all physically meaningful quantities are position independent, the mean density of the ESU must be zero.

\section{Non-Static FRW Universe: Big Bang Model}

In physics, we have various fundamental constants like $G, c, h, m_{e}$ etc. But in no case there is any evidence that the values of such constants directly depend of ambient factors like mean density of universe. In fact, all truly fundamental factors allow themselves to be determined with high precision by judicious combination of theory and experiments. The only exception here is the supposed $\Lambda$ ! This anomaly seems to be resolved with the result that there is no $\Lambda$ ! On the other hand, cosmologists have found that distant Type 1a supernovae appear to be fainter than expected if one would assume 1) them to be standard candles and there is no evolutionary effect in the intrinsic luminosities of the distant supernovae, 2) Assume that cosmological luminosity distance measurements to be perfect; i.e., assume that standard Big Bang Model (BBM) is correct, 3) Assume that there is no dust absorption of lights from distant supernovae. And by ignoring such questions, in the paradigm of the BBM, such extra faintness was interpreted as the proof of existence of $\Lambda$ or "Dark Energy" (In fact, the Physics Nobel for 2011 was due to such an interpretation):

$$
\ddot{S} / S=-\frac{4 \pi}{3}\left(\rho_{e}+3 p_{e}\right)=-\frac{4 \pi}{3}\left(\rho+3 p-\frac{\Lambda}{4 \pi}\right)
$$

The extra faintness is explained by assuming that the galaxies are further away than expected (in a decelerating universe); i.e., by considering $\ddot{S}>0$ and which is possible for a fine tuned positive

$$
\frac{\Lambda}{4 \pi} \geq(\rho+3 p)
$$

But if indeed $\Lambda=0$, then BBM cannot explain a supposed $\ddot{S}>0$. Nonetheless, it has been claimed that if "Cold Dark Matter", another crucial ingredient of BBM, will have pressure, BBM would be able to explain 
$\ddot{S}>0$ even if $\Lambda=0$ [28]. However, this suggestions is patently false because $\rho+3 p$ includes contribution from both visible matter and DM, and any increase in the value of matter pressure $p$, can only give $\ddot{S}<0$. Therefore, the faintness of distant supernovae need not imply that they are further away; and the faintness could be due to unknown systemetic effects [29].

The fact that the BBM cannot explain the physical universe is apparent from the fact, for the BBM [30]

$$
g_{00}=\exp \frac{-2 p_{e}}{p_{e}+\rho_{e}}
$$

And in oder that the FRW metric indeed has $g_{00}=1$, one must have

$$
\frac{-2 p_{e}}{p_{e}+\rho_{e}}=0
$$

Since $\left|p_{e}+\rho_{e}\right|<\infty$, it is necessary here that $p_{e}=p-\Lambda / 8 \pi=0$. Then, it is immediately seen from Equation (134) that, BBM model must (actually) have

$$
\frac{\ddot{S}}{S}=-\frac{4 \pi \rho_{e}}{3} \leq 0
$$

Thus contrary to the popular belief, the BBM actually cannot explain any accelerating universe! Further $p_{e}=p-\Lambda / 8 \pi=0$ would imply $p=\Lambda / 8 \pi$. And if we would rule out such a fine tuning of matter pressure, we should have $p=\Lambda / 8 \pi=0$ for the BBM irrespective of the result obtained in the context of ESU. And with $p=0$, there can be neither any matter pressure nor any microwave background radiation[30].

\section{Why the Big Bang Model Could Be Vacuous}

We have already pointed out that the total energy associated with the FRW metric is zero; and this is not because of any negative self-gravitational interaction. The latter can reduce total energy only when $g_{00}=g_{00}(r, t)<1$, but for the FRW case, $g_{00}=g_{00}(t)=1$ [26,27]. On the other hand field energy density $t_{0}^{0} \geq 0$. Thus vanishing of total energy indicates $\rho_{e}=0$. We probed this important question independently and found that FRW metric should intrinsically correspond to $\rho_{e}=0$ in order that total energy is conserved [31]. In fact there can be a simplified way to confirm this:

Long back Kriele showed that there cannot be any trapped surface for a spherically symmetric homogeneous perfect fluid [32]. This seems to be a special case of the no trapped surface theorem obtained by demanding that timelike worldlines associated with material particles must always remain time like [33], i.e.,

$$
\frac{2 G M(r, t)}{R c^{2}}<1
$$

For the FRW case, one has $R=r S(t)$ and

$$
M(r, t)=\frac{4 \pi R^{3}}{3} \rho_{e}(t)
$$

so that one should have

$$
\frac{8 \pi G}{3 c^{2}} r^{2} \rho_{e}(t) S^{2}(t)<1
$$

And in order that $\rho_{e} S^{2}$ does not blow up either in future or past, one needs to have

$$
\rho_{e}(t) S^{2}(t)=\text { constant }
$$

Again for this, first, it is necessary that $\Lambda=0$ and $\rho_{e}=\rho$ :

$$
\rho(t) S^{2}(t)=\text { constant }
$$

But the FRW model generally obeys $\rho t^{2}=$ constant so that one should have

$$
S(t) \propto t
$$

And it is only for the Milne model (with $k=-1$ ), one obtains $S(t) \propto t$. But the Milne model is empty with $\rho=\rho_{e}=0$ ! Since we have already found $p_{e}=0$, we eventually obtain the vacuum EOS for the FRW metric: $\rho_{e}+p_{e}=\rho+p=0$. Does this result lead to any contradiction with Equation (137)? The answer is "no" because a $0 / 0$ form could be anything including 0 . One may also point out that the result $\rho_{e}=0$ for the FRW model is not rigorous because we did not offer any proof for the usual relation $\rho t^{2}=$ constant. Such an objection would be valid, and thus now we focus on the de-Sitter case with $\rho_{e}=\Lambda / 8 \pi c^{2}$. Now Equation (141) will reduce to

$$
\frac{1}{3} \Lambda r^{2} S^{2}(t)<1
$$

In order that above constraint is satisfied, one must have $\Lambda=0$. Thus, in a rigourous manner, we should have $\Lambda=0$ in order to satisfy the condition of "no trapped surface" in the de-Sitter model.

So for the time being, let us ignore the possibility that the FRW metric subtly represents a vacuum spacetime and which is the reason that there is no density or pressure gradient and there is an universal Newtonian time despite the supposed presence of self-gravity. And even if one would ignore the proof that $\Lambda=0$, there are already many suggestion that $\Lambda$ and "Dark Energy" could be illusions created by an inhomogeneous lumpy universe which is significantly different from the simplistic BBM [34-38]. However if there would be inhomogeneity in a monotonous and continuous manner, there would be a preferred centre of the universe in violation of the "Copernican Principle" of no unique centre. 
On the other hand, the discrete fractal models could still satisfy the Copernican Principle.

\section{Fractal Model}

In a fractal distribution, the number of galaxies increases with $R$ as [39-41]

$$
N(<R) \sim R^{D}
$$

and the number density of galaxies varies as

$$
n(<R) \sim R^{D-3}
$$

Thus, in principle, for a fractal distribution over sufficiently large scale, one may have $n \rightarrow 0, \rho \rightarrow 0$. Most of the fractal patterns however tend to have a preferred center and thus not suitable for "Copernican Principle". But there could be some fractal structures like Levy Dust which may not have any preferred center.

Indeed many cosmologists believe that within the patch of the observed universe, galaxies are distributed in fractal like pattern atleast on scales $\sim 10$ Mpc [42-45], and one may recall here a succint comment by $\mathrm{Wu}, \mathrm{La}-$ hav \& Rees [45]

"The universe is inhomogeneous-and essentially fractal-on the scale of galaxies and clusters of galaxies, but most cosmologists believe that on larger scales it becomes isotropic and homogeneous."

This review written in 1999 however concluded that galaxy distribution is homogeneous on larger scales. But now it is seen that galaxies are distributed in a roughly fractal pattern with dimension $D \sim 2.2$ even on $\sim 100$ Mpc scale [46,48-52]. Note, many of the objections against the fractal model have already been addressed to [53].

\section{Conclusions}

Since cosmological constant $\Lambda=0$, so-called "Dark Energy" too is expected to be absent. Simultaneously it has been found that the Big Bang Model intrinsically corresponds to zero pressure and zero temperature [30]. Note this result is in perfect agreement with the fact that an ideal Hubble flow implies smooth radial motion with no collision amongst the test particles. Thus even if one would assume $\Lambda=0$, in view of Equation (138), the BBM cannot explain any cosmic acceleration. Note, Equation (138) can be obtained by purely Newtonian gravity! But when can a GR result be exactly synonymous with a purely Newtonian result? To appreciate this, have a close look at GR Poission equation (124).

- Assume gravitation is extremely weak:

$$
g_{00} \approx 1+2 \frac{\psi}{c^{2}}
$$

where $\psi$ is the Newtonian potential and $2 \psi / c^{2} \ll 1$. Then by further stting $p=0$, one can obtain the approximate Newtonian Poission equation:

$$
\nabla^{2} \psi \approx 4 \pi G \rho
$$

But by starting from GR equation, one would never obtain an exact Poission's Equation $\nabla^{2} \psi=4 \pi G \rho$ if $\psi \neq 0$. Thus, GR can yield an exact Newtonian result only when the gravitational potential $\psi=0$ ! This suggests that though, mathematically, one can conceive of global clock synchronization and set $g_{00}=1$, physically, gravitation tends to manisfest itself with non-synchronization of clocks! In other words, a model assuming global synchronization of clocks could be vacuous with zero matter density! Thus one expects all dust models to correspond to $\rho=0$. And indeed, the famous Oppenheimer-Snyder dust collapse has explicitly been found to correspond to $\rho=0$ [54]. The Schwarzschild "Black Hole" exact solution too is illusory because the integration constant involved there is actually zero implying true black holes have zero gravitational mass; and the so-called "Black Hole Candidates" must be something else[55]. Similarly, it has been found that a uniform density sphere cannot undergo any adiabatic collapse at all and hence scores of exact solutions indicating collapse must actually correspond to $\rho=0$ [56]. In general, there cannot be any adiabatic gravitational evolution at all, and which shows that all exact solutions indicating evolution must tacitly correspond to $\rho=0$ [26]. Even the famous interior solution for a static uniform density spherical star is illusory in the sense that it actually corresponds to $\rho=0$ [18]. This may be suggesting that self-gravity manifests itself not only through pressure gradient and non-syncronization of clocks, but also through density inhomogeneity.

Thus it is indeed possible that FRW metric too subtly corresponds to a vacuum solution just like its static counterpart. In general, though, "exact GR solutions" may often be physically meaningful for the static cases, they need not be so for the complex non-static cases; the complexities of a physical system, such as unknown and evolving equation of state of matter, unknown radiation transport properties, unknown evolution of shear and dissipation, may rarely allow physically meaningful non-static exact solutions. For instance, it has been found that celebrated non-static Kerr solution is an illusion because the integration constants, namely the rotation parameter $a$ and mass $m$ are actually zero: $a=m=0$ [57,58].

Even if this suggestion that FRW metric too is tacitly a vacuum solution, would be ignored at this moment, there is now firm conclusion that galaxies are distributed in a fractal pattern atleast on scales $\sim 100 \mathrm{Mpc}$ in violation of 
the assumptions of $\Lambda$ CDM model [46-52]. In fact the latest Sloan Digital Sky Survey results confirm that the observed universe is indeed lumpy on the largest scale [59], and Thomas et al. too have rightly questioned the physical reality of "Dark Energy" [59]. The recent observation that there is no hint of non-baryonic "Cold Dark Matter" in two dwarf galaxies Fornax and Sculptor raises serious questions about the validity of the $\Lambda$ CDM model [60].

Note that it is possible that the distant Type 1a supernovae may have different luminosities compared to local ones because of different metallicities or other evolutionary effects. And even if they would be standard candles without appreciable cosmic evolution, there is some chance that their faintness could be due to opacities of distant inter-galactic medium. For example, it has been pointed out that Lyman Alpha clouds might introduce some non-transparency for optical emission from distant universe [61]. It has been also argued that the atmosphere of planets could be additional sources of opacities for very distant supernovae lights [62]. Also, there could be a fundamental non-accuracy in estimating the precise luminosity distances of distant supernovae because of missing gaps and extrapolations in the cosmic distance measurement ladders. Further, consider the fact that we are able to "see" galaxies and measure their redshifts because they are radiating. But in the standard cosmologies, galaxies are considered as non-radiating neutral "dust" particle. Consequently, one should leave here an open window for some hitherto unknown physics or surprise for understanding this new phenomenon. In general, there could be unknown systemetic effect behind the interpretation of extra faintness of distant Type 1a supernovae [29].

And if the universe would indeed be unbounded and infinite hierarchial fractal, then the basic assumption behind the formulation of Hubble's law, i.e., strict homogeneity, gets invalidated. In such a case, the universe need not be expanding and the observed redshits could be of non-Doppler origin. Recall long back Ellis [63] argued that observations really cannot rule out a static but inhomogeneous universe. Photons propagating over cosmic scales might undergo energy losses by yet unknown feeble quantum electrodynamical interaction with the quantum vacuum [64]. Photons might also lose energy by interacting with plasma permeating the whole cosmos [65]. It is interesting to note that the plasma redshift proposed by Ari Brynjolfsson can simulate a value of "Hubble Parameter" $\sim 75 \mathrm{Km} / \mathrm{s} / \mathrm{Mpc}$ by considering a mean cosmic free electron density of $\sim 0.0002 \mathrm{~cm}^{-3}$ [64-68]. The only "new physics" involved with his redshift mechanism is to include an electron-electron collision term in an otherwise standard quantum derivation of photon-electron plasma interaction cross-sections. Thus, the excess dimming at high redshifts could simply be a consequence of the Compton scattering that accompanies the redshifting mechanism in the hot, sparse electron plasmas that fill intergalactic space.

In Brynjolfsson's cosmology, the universe is not expanding, and there is no time dilation affecting supernova light curves, and it claims to account for the redshift/SNe Ia luminosity data.

Though the BBM has had many successes to its credit and it is the most developed cosmology, it fails to satisfactory explain many cosmological facts [69]. And irrespective of the presence or absence of an appropriate static model for the universe, there are innumerable problems with the BBM. In particular,

- BBM may be failing the angular size test; whereas a static model fits data better [70].

- The age of extremely red and massive galaxies at very high redshift may be contradicting the $\Lambda \mathrm{CDM}$ cosmology [71]. And most importantly,

- The Gamma Ray Bursts having zs as large as $\sim 9$ do not show signs of supposed space-expansion related time dilation $[72,73]$.

In fact a static model of universe (which must be entirely different from a vacuous ESU) may fit at least some of the cosmological observations better $[74,75]$ without any exotic assumptions.

Also, in an infinite and eternal universe, part of the starlight may get thermalized to generate the observed microwave background radiation, as was first suggested by Sir Fred Hoyle. However, there could also be alternative explanations for the same. For instance, the microwave background radition might be due to superposition of redshifted quiescent surface glow of the Eternally Collapsing Objects, the so-called "Black Holes" [76].

Note, when acoustic wave packets traverse a dispersive medium for very long duration, they develop "redshift" [77]. And if the wave packet will have a Gaussian shape, it maintains this shape despite central energy loss; and further, the attendant redshift obeys the Hubble's Law [77]. Recall, all emitted electromagnetic lines are actually narrow "wave packets", and their propagation in the cosmos is likely to be dissipative. At a classical level, one can think that the cosmos with matter and plasma behaves as a "dissipative medium"; and on a Quantum Electrodynamic level, one may take the QED vacuum as a "dissipative medium". Can the electromagnetic wave packets too undergo red-shift similar to the acoustic waves? Or can the electromagnetic wave packets spread in the momentum space like the "de-Broglie matter waves" and somehow simulate the Hubble's law?

In any case, from a purely observational view point, one can say that [78] "it is impossible to conclude either 
way whether the Universe is expanding or static. The evidence is equivocal; open to more than one interpretation. It would seem that cosmology is far from a precision science, and there is still a lot more work that needs to be done to resolve the apparent evidences."

\section{Endnote}

A very old version of this paper was submitted to Phys. Rev. D. in 2008. And though the assigned referee did not point out any technical error, he would not accept the result $\Lambda=0$ claiming it would be against observation! Simulataneously one moderator of arXiv.org also quietly shifted it from astro-ph to phys-gen section; and in frustration, I did not look at this manuscript for 3 years. Only this yesr, another version was submitted to J. Cosmology $\&$ Astroparticle Physics. The referee here accepted that the proofs claiming $\Lambda=0$ are correct. But he suggested that the revised version should (1) not only cite and highlight the reference [28] for explaining the $\Lambda=0$ result, but (2) all explanations in the light of inhomogeneity, fractal strcture etc. should be avoided. While the revised version conceded the first part of this arm-twisting, it could not yield to the latter part. Then this paper was rejected by JCAP even after accepting that the proof $\Lambda=0$ was correct. Note here that the suggestion by Kleidis \& Spyrou that an increase in the value of $p$ for the DM can make $\ddot{S}>0$ even when $\Lambda=0$ is completely wrong (see Equation [134]) even though it is published in A \& A! Yet Dr Spyrou killed this paper in order to highlight his completely wrong idea by suppressing all competing correct ideas! However, the referee of IJAA found this paper so important that the processing charges were kindly waived off. Thanks IJAA.

\section{Reference}

[1] S. Weinberg, "Gravitation and Cosmology: Principles and Applications of General Theory of Relativity," John Wiley, New York, 1972.

[2] L. D. Landau and E. M. Lifshitz, "Classical Theory of Fields," Pergamon Press, Oxford, 1962.

[3] R. C. Tolman, "Static Solutions of Einstein's Field Equations for Spheres of Fluid," Physical Review, Vol. 55, No. 4, 1939, pp. 364-373

[4] R. C. Tolman, "Relativity, Thermodynamics \& Cosmology," Clarendon Press, Oxford, 1962.

[5] H. Nariai, "On a New Cosmological Solution of Einstein's Field Equations of Gravitation, “ General Relativity and Gravitation, Vol. 31, No. 6, 1999, pp. 963-971. doi:10.1023/A:1026602724948

[6] Z. Stuchlik and S. Hledk, "Some Properties of the Schwarzschild-de Sitter and Schwarzschild-anti-de Sitter Spacetimes," Physical Review D, Vol. 60, No. 4, 2000, Article
ID: 044006.

[7] C. G. Boehmer, "General Relativistic Static Fluid Solutions with Cosmological Constant," Diploma Thesis, Potsdam University, Potsdam, 2002, gr-qc/0308057.

[8] C. G. Bohmer and G. Fodor, "Perfect Fluid Spheres with Cosmological Constant," Physical Review D, Vol. 77, No. 6, 2008, Article ID: 064008

[9] A. D. Rendall and B. G. Schmidt, "Existence and Properties of Spherically Symmetric Static Fluid Bodies with a Given Equation of State," Classical \& Quantum Gravity, Vol. 8, No. 5, 1991, p. 985. doi:10.1088/0264-9381/8/5/022

[10] T. W. Baumgarte and A. D. Rendall, "Regularity of Spherically Symmetric Static Solutions of the Einstein Equations," Classical \& Quantum Gravity, Vol. 10, No. 2, 1993, p. 327. doi:10.1088/0264-9381/10/2/014

[11] A. Einstein, "On a Stationary System with Spherical Symmetry Consisting of Many Gravitating Masses," Annals of Mathematics, Vol. 40, No. 4, 1939, pp. 922-936. doi: $10.2307 / 1968902$

[12] P. S. Florides, "A New Interior Schwarzschild Solution," Proceedings of the Royal Society of London A, Mathematical and Physical Sciences, Vol. 337, No. 1611, 1974, pp. 529-535.

[13] N. K. Kofinti, "On a New Interior Schwarzschild Solution," General Relativity and Gravitation, Vol. 17, No. 3, 1985, pp. 245-249. doi:10.1007/BF00760246

[14] C. G. Bohmer and F. S. N. Lobo, "A New Two-Sphere Singularity in General Relativity," International Journal of Modern Physics D, Vol. 17, No. 06, 2008, pp. 897-910. doi:10.1142/S0218271808012565

[15] L. Rudnick, S. Brown and L. R. Williams, "Extragalactic Radio Sources and the WMAP Cold Spot," The Astrophysical Journal, Vol. 671, No. 1, 2007, pp. 40-44.

[16] C. Park, et al., "Topology Analysis of the Sloan Digital Sky Survey. I. Scale and Luminosity Dependence," The Astrophysical Journal, Vol. 633, No. 1, 2005, pp. 11-22. doi:10.1086/452625

[17] R. R. Caldwell \& A. Stebbins, "A Test of the Copernican Principle," Physical Review Letters, Vol. 100, No. 19, 2008, Article ID: 191302.

[18] A. Mitra, "No Uniform Density Star in General Relativity," Astrophysics and Space Science, Vol. 333, No. 1, 2010, pp. 169-174. doi:10.1007/s10509-010-0567-8

[19] H. A. Buchdahl, "General Relativistic Fluid Spheres," Physical Review, Vol. 116, No. 4, 1959, pp. 1027-1034. doi:10.1103/PhysRev.116.1027

[20] N. Rosen, "The Energy of the Universe," General Relativity and Gravitation, Vol. 26, No. 3, 1994, pp. 319-321. doi:10.1007/BF02108013

[21] F. I. Cooperstock, "Perspectives on the energy of the universe," General Relativity and Gravitation, Vol. 26, No. 3, 1994, pp. 323-327. doi:10.1007/BF02108014

[22] V. B. Johri, D. Kalligas, G. P. Singh and C. W. F. Everitt, "Gravitational Energy in the Expanding Universe," General Relativity and Gravitation, Vol. 27, No. 3, 1995, pp. 


\section{3-318. doi:10.1007/BF02109127}

[23] N. Banerjee and N. S. Sen, "Einstein Pseudotensor and Total Energy of the Universe," Pramana, Vol. 49, No. 6, 1997, pp. 609-615. doi:10.1007/BF02848334

[24] S. S. Xulu, "Total Energy of the Bianchi Type I Universes," International Journal of Theoretical Physics, Vol. 39, No. 4, 2000, pp. 1153-1161. doi:10.1023/A:1003670928681

[25] V. Faraoni and F. I. Cooperstock, "On the Total Energy of Open Friedmann-Robertson-Walker Universes," The Astrophysical Journal, Vol. 587, No. 2, 2003, pp. 483486. doi: $10.1086 / 368258$

[26] A. Mitra, "Why Gravitational Contraction Must Be Accompanied by Emission of Radiation in Both Newtonian and Einstein Gravity," Physical Review D, Vol. 74, No. 2, 2006, Article ID: 024010.

[27] A. Mitra, "Does Pressure Increase or Decrease Active Gravitational Mass Density," Physics Letters B, Vol. 685, No. 1, 2010, pp. 8-11.

[28] K. Kleidis and N. K. Spyrou, "A Conventional Approach to the Dark-Energy Concept," Astronomy \& Astrophysics, Vol. 529, No. 1, 2011, Article ID: 529A26.

[29] S. D. M. White, "Fundamentalist Physics: Why Dark Energy Is Bad for Astronomy," Reports on Progress in Physics, Vol. 70, No. 6, 2007, pp. 883-897.

[30] A. Mitra, "The Matter in the Big-Bang Model Is Dust and Not Any Arbitrary Perfect Fluid!” Astrophysics and Space Science, Vol. 333, No. 1, 2011, pp. 351-356. doi:10.1007/s10509-011-0635-8

[31] A. Mitra, "Einstein Energy Associated with the FriedmannRobertson-Walker Metric," General Relativity and Gravitation, Vol. 42, No. 3, 2010, pp. 443-469,

[32] M. Kriele, "A Bound on the Concentration of Matter in Spherically Symmetric Stars \& Its Application for the Existence of Black-Holes," Rendiconti del Seminario Matematico (Università e Politecnico di Torino), Vol. 50, No. 1, 1992, pp. 147-152.

[33] A. Mitra, "Quantum Information Paradox: Real or Fictitious," Pramana, Vol. 73, No. 3, 2009, pp. 615-620.

[34] E. W. Kolb, "Backreaction of Inhomogeneities Can Mimic Dark Energy," Classical and Quantum Gravity, Vol. 28, No. 16, 2011, Article ID: 164009. doi:10.1088/0264-9381/28/16/164009

[35] B. M. Leith, S. C. C. Ng and D. L. Wiltshire, "Gravitational Energy as Dark Energy: Concordance of Cosmological Tests," The Astrophysical Journal, Vol. 672, No. 2, 2008, pp. L91-L94. doi:10.1086/527034

[36] C. Clarkson and R. Maartens, "Inhomogeneity and the Foundations of Concordance Cosmology," Classical and Quantum Gravity, Vol. 27, No. 12, 2010, Article ID: 124008.

[37] M. Regis and C. Clarkson, "The Cosmic Microwave Background in an Inhomogeneous Universe," Journal of Cosmology and Astroparticle Physics, Vol. 2011, No. 2, 2011, Article ID: 013.

[38] C. Tsagas, "Peculiar Motions, Accelerated Expansion, and the Cosmological Axis," Physical Review D, Vol. 84, No. 6, 2011, Article ID: 063503.
[39] B. B. Mandelbrot, “The Fractal Geometry of Nature," Freeman, New York, 1982.

[40] Y. Baryshev and P. Teerikopri, "Discovery of Cosmic Fractals," World Scientific, Singapore, 2002. doi: $10.1142 / 9789812388780$

[41] C. V. L. Charlier, "On the Structure of the Universe," Publications of the Astronomical Society of the Pacific, Vol. 37, 1925, pp. 53-76. doi:10.1086/123477

[42] G. de Vaucouleurs, "The Case for a Hierarchical Cosmology," Science, Vol. 167, No. 3922, 1970, pp. 1203-1213. doi:10.1126/science.167.3922.1203

[43] Yu. V. Baryshev, F. Sylos Labini, M. Montuori, L. Pietronero and P. Teerikorpi, "On the Fractal Structure of Galaxy Distribution and Its Implications for Cosmology," Fractals, Vol. 6, No. 3, 1998, pp. 231-243. doi:10.1142/S0218348X98000286

[44] V. Martinez, "COSMOLOGY: Is the Universe Fractal?" Science, Vol. 285, No. 5413, 1999, p. 445-446. doi:10.1126/science.284.5413.445

[45] K. Wu, O. Lahav and M. Rees, "The Large-Scale Smoothness of the Universe," Nature, Vol. 397, No. 6716, 1999, pp. 225-230. doi:10.1038/16637

[46] F. Sylos Labini, N. L. Vasilyev and Yu. V. Baryshev, “Absence of Anti-Correlations and of Baryon Acoustic Oscillations in the Galaxy Correlation Function from the Sloan Digital Sky Survey Data Release 7," Astronomy and Astrophysics, Vol. 505, No. 3, 2009, pp. 981-990. doi:10.1051/0004-6361/200911987

[47] F. Sylos Labini, N. L. Vasilyev and Yu. V. Baryshev, "Absence of Self-Averaging and of Homogeneity in the Large-Scale Galaxy Distribution," Europhysics Letters, Vol. 86, No. 4, 2009, Article ID: 49001. doi:10.1209/0295-5075/86/49001

[48] F. Sylos Labini, N. L. Vasilyev, L. Pietronero and Yu. V. Baryshev, "Persistent Fluctuations in the Distribution of Galaxies from the Two-degree Field Galaxy Redshift Survey," Europhysics Letters, Vol. 85, No. 2, 2009, Article ID: 29002. doi:10.1209/0295-5075/85/29002

[49] F. Sylos Labini and Yu. V. Baryshev, "Testing the Copernican and Cosmological Principles in the Local Universe with Galaxy Surveys," Journal of Cosmology and Astroparticle Physics, Vol. 2010, No. 6, 2010, Article ID: 021.

[50] F. Sylos Labini and L. Pietronero, "The Complex Universe: Recent Observations and Theoretical Challenges," Journal of Statistical Mechanics: Theory and Experiment, Vol. 2010, No. 11, 2010, Article ID: 11029

[51] F. Sylos Labini, "Inhomogeneities in the Universe," Classical and Quantum Gravity, Vol. 28, No. 16, 2011, Article ID: 164003.

[52] F. Sylos Labini, "Very Large Scale Correlations in the Galaxy Distribution," Europhysics Letters, Vol. 96, No. 5, 2011, Article ID: 59001. doi:10.1007/s10509-010-0578-5

[53] Y. Baryshev and P. Teerikopri, "Fractal Approach to LargeScale Galaxy Distribution," Russian Academy of Sciences, Vol. 59, 2005. arXiv.org:astro-ph/0505185

[54] A. Mitra, "The fallacy of Oppenheimer Snyder Collapse: 
No General Relativistic Collapse at All, No Black Hole, No Physical Singularity," Astrophysics and Space Science, Vol. 332, No. 1, 2011 pp. 43-48.

[55] A. Mitra, "Why the Astrophysical Black Hole Candidates Are Not Rotating Black Holes," 2004 arXiv:astro-ph/0407501

[56] A. Mitra, "Why the Astrophysical Black Hole Candidates May Not Be Black Holes at All,” 2004. arXiv:astro-ph/0409049

[57] A. Mitra, "Comments on 'The Euclidean Gravitational Action as Black Hole Entropy, Singularities, and SpaceTime Voids'," Journal of Mathematical Physics, Vol. 50, No. 4, 2009, Article ID: 042502.

[58] A. Mitra, "Revisiting the Old Problem of General Relativistic Adiabatic Collapse of a Uniform Density Self-Gravitating Sphere," Gravitation \& Cosmology, Vol. 18, No. 1, 2012, (To be published).

[59] S. A. Thomas, F. B. Abdalla and O. Lahav, "Excess Clustering on Large Scales in the MegaZ DR7 Photometric Redshift Survey," Physical Review Letters, Vol. 106, No. 24, 2011, Article ID: 241301.

[60] M. G. Walker, C. Combet, J. A. Hinton, D. Maurin and D. M. I. Wilkinson, "A Method for Measuring (Slopes of) the Mass Profiles of Dwarf Spheroidal Galaxies," Astrophysical Journal Letters, Vol. 742, No. 1, 2011, Article ID: 20(2011).

[61] R. E. Schild \& M. Dekker, "The Transparency of the Universe limited by Lyman Alpha clouds," Astronomische Nachrichten, Vol. 327, No. 7, 2006, pp.729-732. doi:10.1002/asna.200510612

[62] C. H. Gibson and R. E. Schild, "Planets and Dark Energy," Proceedings of the International Conference on Problems in Practical Cosmology, St. Petersburg, 23-27 June 2008, pp. 232-238.

[63] G. R. F. Ellis, "Is the Universe Expanding?" General Relativity and Gravitation, Vol. 9, No. 2, 1978, pp. 87-94. doi:10.1007/BF00760145

[64] Y. A. Yatsunenk and J. A. Budagov, "Red Shift in a Laboratory Environment," 2011. arXiv:1103.0808
[65] A. Brynjolfsson, "Hubble Constant from Lensing in Plasma-Redshift Cosmology, and Intrinsic Redshift of Quasars," 2004. arXiv:astro-ph/0411666

[66] A. Brynjolfsson, "The Type Ia Supernovae and the Hubble's Constant", 2004. arXiv:astro-ph/0407430

[67] A. Brynjolfsson, "Magnitude-Redshift Relation for SNe Ia, Time Dilation, and Plasma Redshift," 2006. arXiv:astro-ph/0602500

[68] A. Brynjolfsson, "Surface Brightness in Plasma-Redshift Cosmology,” 2006. arXiv:astro-ph/0605599

[69] Ashwini Kumar Lal, "Big Bang? A Critical Review," Journal of Cosmology, Vol. 6, 2010, pp. 1533-1547.

[70] M. Lopez-Corredoira, "Angular Size Test on the Expansion of the Universe," International Journal of Modern Physics D, Vol. 19, No. 3, 2010, pp. 245-291.

[71] N. Castro-Rodriguez and M. Lopez-Corredoira, "The Age of Extremely Red and Massive Galaxies at Very High Redshift," Astronomy \& Astrophysics, 2011 (in press).

[72] D. Kocevski and V. Petrosian, "On the Lack of Time Dilation Signatures in Gamma-ray Burst Light Curves," Submitted to The Astrophysical Journal, 2011.

[73] D. F. Crawford, "No Evidence of Time Dilation in GammaRay Burst Data," 2009. arXiv:0901.4169

[74] D. F. Crawford, "Observational Evidence Favors a Static Universe (Part I)," Journal of Cosmology, Vol. 13, 2011, pp. $3875-3946$

[75] D. F. Crawford, "Observations of Type Ia Supernovae Are Consistent with a Static Universe," 2009. arXiv:0901.4172

[76] A. Mitra, "Cosmological Properties of Eternally Collapsing Objects (ECOs)," Proceedings of International Conference on Problems of Practical Cosmology, Vol. 1, St. Petersburg, 23-27 June 2008, pp. 304-313.

[77] C. I. Christov, "On the Evolution of Localized Wave Packets Governed by a Dissipative Wave Equation," Wave Motion, Vol. 45, No. 3, 2008, pp. 154-161. doi:10.1016/j.wavemoti.2007.05.004

[78] J. G. Hartnett, "Is the Universe Really Expanding?" 2011. arXiv:1107.24851 